\title{
Rezension zu: Takeshi Amemiya, Economy and Economics of Ancient Greece (London 2007)
}

\author{
Nicolas Krocker
}

Die Wirtschaft des antiken Griechenlands wirkt aufgrund der schwierigen Quellenlage sowie ihrer Andersartigkeit und Komplexität auf ihren Betrachter in manchen Bereichen wie ein „enigma“. ${ }^{1}$ Ihren Ursprung hatte die Forschungsgeschichte zur antiken Wirtschaft im ausgehenden 19. Jahrhundert mit der Bücher-Meyer-Kontroverse, aus der sich anschließend die beiden konkurrierenden Schulen der „Primitivisten“ sowie „Modernisten“ entwickelten. ${ }^{2}$ Bis in die 1970er Jahre war die modernistische Sichtweise, geprägt durch die Arbeiten von Michael Rostovtzeff und William Westermann, die vorherrschende Lehrmeinung. Beeinflusst von Max Weber und Karl Polanyi entwarf Moses I. Finley in seinem 1973 erschienenen Werk „The Ancient Economy“ ein (neo-)primitivistisches Gegenkonzept, welches von seinen Befürwortern als „new orthodoxy“ bezeichnet wurde. ${ }^{3}$ In der neuesten Literatur kann zum Teil eine Hinwendung zu einer formalistischen Methode der Untersuchung der griechisch-römischen Ökonomie konstatiert werden, da die wirtschaftshistorischen Positionen von Douglass North, einem der Hauptvertreter der Neuen Institutionenökonomik (NIÖ bzw. NIE), in den Vordergrund rücken. ${ }^{4}$ Während Formalisten von einer universellen Gültigkeit der neoklassischen Wirtschaftstheorie ausgehen, negieren Befürworter des Substantivismus die Anwendbarkeit dieses Theoriegebäudes auf nicht-westliche und vormoderne Gesellschaften, deren Wirtschaftssysteme nicht kapitalistischen Ursprungs sind. ${ }^{5}$

Takeshi Amemiya, Professor für Volkswirtschaft an der Stanford Universität, steuert mit seinen Lehrbüchern einen wichtigen Bestandteil zur ökonometrischen Ausbildung bei. ${ }^{6}$ Seine weiteren Forschungsschwerpunkte liegen in der Wirtschaftsgeschichte des antiken Griechenland sowie der griechischen Philosophie. ${ }^{7}$ Somit gehört er der kleinen Gruppe von Wissenschaftlern an, welche Wirtschaftswissenschaftler und Althistoriker in einer Person und folglich auf beiden

\footnotetext{
${ }^{1}$ Vgl. D. T. Engen, The economy of ancient Greece, in: R. Whaples (Hg.), EH.Net Encyclopedia, S. 125, hier S. 1, (http://eh.net/encyclopedia/article/engen.greece), abgerufen am 18. Dezember 2009.

${ }^{2}$ Vgl. K. Strobel, Zwischen Primitivismus und Modernismus: Die römische Keramikindustrie und die Suche nach einem Kategorisierungsmodell der römischen Wirtschaft. Eine Einführung, in: Ders. (Hg.), Forschungen zur römischen Keramikindustrie. Produktions-, Rechts- und Distributionsstrukturen. Akten des 1. Trierer Symposiums zur antiken Wirtschaftsgeschichte (Trierer historische Forschungen 42), Mainz 2000, S. 1-8, hier S. 1.

${ }^{3}$ Vgl. dazu zuletzt H.-J. Drexhage, H. Konen, K. Ruffing, Die Wirtschaft der römischen Kaiserzeit in der modernen Deutung: Einige Überlegungen, in: K. Strobel (Hg.), Die Ökonomie des Imperium Romanum. Strukturen, Modelle und Wertungen im Spannungsfeld von Modernismus und Neoprimitivismus. Akten des 3. Trierer Symposiums zur Antiken Wirtschaftsgeschichte (Pharos XVII), St. Katharinen 2002, S. 1-66, hier S. 1.

${ }^{4}$ Vgl. I. Morris, R. P. Saller, W. Scheidel, Introduction, in: Dies. (Hg.), The Cambridge economic history of the Greco-Roman world, Cambridge 2007, S. 1-12, bes. S. 1 f., 7 u. 12.

${ }^{5}$ Vgl. N. Morley, Theories, models and concepts in ancient history, London 2004, S. 43.

6 T. Amemiya, Advanced econometrics, Oxford 1986; Ders., Introduction to statistics and econometrics, Cambridge, Mass. 1994.

${ }^{7}$ Vgl. J. L. Powell, THE ET INTERVIEW: TAKESHI AMEMIYA. Interviewed by James L. Powell, in: Econometric Theory 23 (2007), S. 155-181, hier S. 156-160.
} 
Gebieten mit der Fachterminologie sowie Methodik vertraut sind. ${ }^{8}$ Mit seinem Buch „Economy and Economics of Ancient Greece“ erscheint ein weiterer Titel zu einem in den letzten Jahren noch immer stark bearbeiteten Forschungsfeld der Alten Geschichte in der Post-Finley Ära. ${ }^{9}$

$\mathrm{Zu}$ Beginn des Buches findet sich eine kurze Einführung in die griechische Geschichte von der mykenischen bis zur klassischen Zeit. Darauf folgt eine genaue Untersuchung der athenischen Gesellschaft und Wirtschaft des 5. und 4. Jahrhunderts v. Chr.. Den Abschluss bildet eine Darstellung der ökonomischen Gedankengänge von Xenophon, Platon und Aristoteles. Der Verfasser setzt sich zum Ziel unter Hinzuziehung vielfältiger literarischer und epigraphischer Quellen ein in sich stimmiges Gesamtmodell der athenischen Wirtschaft des 4. Jahrhunderts zu entwickeln und folgt damit der in der neueren Forschung postulierten Forderung nach einer stärkeren Theoretisierung der antiken Wirtschaftsgeschichte und der Verbindung von empirischen Fakten mit Modellen. ${ }^{10}$

Der erste Teil „History, Society, Culture“ beginnt mit einem historischen Überblick über die einzelnen griechischen Epochen, wobei die klassische und hellenistische Epoche in chronologischer Reihenfolge schlagwortartig abgehandelt werden. Anschließend erfolgt ein Vergleich zwischen der griechischen Religion und der in Japan praktizierten Shinto-Religion, gefolgt von kurzen Abschnitten zu den Begriffen „Ehre“, „Schande“, „Oikos“, „Polis“, „Freundschaftsdiensten“, „Wettbewerb“, „Wohlstand“, „Armut“, „Theater“, „Kunst“, „Erziehung“, „Philosophie“ sowie „Wissenschaft“. Dabei erscheint die Argumentation teilweise, etwa bei der Frage nach der sozialen Stellung der Frau, nicht unbedingt stringent; teilweise werden auch Themenkomplexe, wie etwa „Bürgerrecht“, „Ehe“ und „Erbschaft“, nur sehr kursorisch abgehandelt. Nach einem Versuch, die Größe der Sklavenpopulation zu schätzen, werden die verschiedenen Beschäftigungsverhältnisse von Sklaven aufgezeigt, deren Bannbreite vom abgabenpflichtigen Betreiber einer Werkstatt über Haus- und Ackerbausklaven bis hin zu Bergbausklaven reichen konnte. Bei der Thematisierung der Anzahl der Sklavenbesitzer in der athenischen Gesellschaft wirft der Autor einige interessante Fragen, wie etwa diejenige nach dem Wechselverhältnis von Sklavenwirtschaft und technischem Fortschritt sowie diejenige nach der Sklavenwirtschaft als einer Voraussetzung für das Funktionieren der athenischen Demokratie, auf. Doch fällt auch in diesen Punkten die Intensität der Diskussion bisweilen etwas zu knapp aus. Ausgehend von den bei Demosthenes überlieferten Angaben werden Sklavenpreise sowie die Produktivität der Sklaven berechnet. Unter Zuhilfenahme weiterer Texte von Aischines, Demosthenes und Lysias schließt sich daran eine Tabelle mit der Größe von Handwerksbetrieben, ihren

\footnotetext{
${ }^{8}$ So etwa auch Peter Temin; Vgl. P. Temin, A Market Economy in the Early Roman Empire, in: JRS 91 (2001), S. 169-181; vgl. Ders., The Economy of the Early Roman Empire, in: Journal of Economic Perspectives 20 (2006), S. 133-151.

9 Z. H. Archibald, J. Davies u. a. (Hg.), Hellenistic Economies, London 2001; P. Cartledge, E. E. Cohen u. a. (Hg.), Money, Labour and Land. Approaches to the economics of ancient Greece, London 2002; A. Eich, Die politische Ökonomie des antiken Griechenland. (6. - 3. Jahrhundert v. Chr.), Köln 2006; U. Fellmeth, Pecunia non olet. Die Wirtschaft der antiken Welt, Darmstadt 2008; J. G. Manning, I. Morris (Hg.), The ancient economy. Evidence and Models, Stanford 2005; D. M. Mattingly, J. Salmon (Hg.), Economies beyond agriculture in the classical world, London 2001; A. Moreno, Feeding the democracy. The Athenian grain supply in the fifth and fourth centuries BC, New York 2007; I. Morris, R. P. Saller, W. Scheidel (Hg.), The Cambridge economic history of the Greco-Roman world, Cambridge 2007; W. Scheidel, S. v. Reden (Hg.), The ancient economy, Edinburgh 2002.

${ }^{10}$ Vgl. I. Morris, J .G. Manning, Introduction, in: Dies. (Hg.), The Ancient Economy. Evidence and Models, Stanford 2005, S. 1-44, hier S. 2.
} 
Produkten, Jahreseinkommen sowie Organisationsformen an. Der zwischen diesem Teil und der Diskussion der demographischen Entwicklung der athenischen Gesamtbevölkerung eingeschobene Absatz über Homosexualität im antiken Griechenland verwundert hingegen ein wenig und wäre an anderer Stelle besser platziert gewesen. Im folgenden Abschnitt erfolgt eine Schilderung der wichtigsten Institutionen der athenischen Demokratie. Den Abschluss des ersten Teils bildet die Frage, ob die Demokratie in Athen ein Erfolg war oder nicht. Zu diesem Zweck werden Beispiele für Erfolge und Misserfolge gegenübergestellt und anschließend das komplexe Wechselspiel zwischen Elite und Masse thematisiert.

Der zweite Teil „Economy“ bildet den interessantesten Part des Buches. Amemiya beginnt mit einer prägnanten und inhaltsreichen Darstellung der Debatte zwischen Modernisten und Primitivisten bzw. Formalisten und Substantivisten. ${ }^{11}$ Die immer noch zentrale Bedeutung von Moses Finely's substantivistischer Thesen für ein Verständnis der athenischen Geschichte, wie sie Ian Morris bereits 1994 konstatierte, zeigt sich darin, dass sich auch Amemiya intensiv mit dessen Thesen auseinandersetzt. ${ }^{12}$ Jedoch kommt der Autor zu dem Schluss, „that the economy of this period [5. und 4. Jahrhundert] was developed in considerable degree in terms of the market, manufacturing process, and financial institutions” (S. 60). Aufgabe der nächsten Kapitel sei es, diesen Punkt genauer zu elaborieren.

Unter der Prämisse eines effizient arbeitenden Marktes, folgen ausführliche Listen von Preisen für unterschiedlichste Güter, Löhne, agrarische Erzeugnisse und deren Konsum. Daran schließt sich ein Abschnitt über das Ausmaß, die Bedeutung und die Rahmenbedingungen des attischen Handels an, der seinen Abschluss in einem auf fünf Seiten dargelegten hypothetisch-mathematischen Modell über den jährlichen athenischen Sklavenimport findet. Ähnlich verfährt Amemiya bei dem Themenkomplex „Öffentliche Finanzen“, indem er in Form von ausführlichen Listen Einnahmen und Ausgaben des athenischen Staates darstellt. Es folgt ein Kapitel über das Geld- und Kreditwesen. ${ }^{13}$ Nun entwirft der Autor sein Modell der athenischen Wirtschaft des 4. Jahrhunderts: Es werden fünf Bilanzgleichungen, welche die Einkünfte und Ausgaben der Sektoren „Arm“, „Reich“, „Handel- und Gewerbetreibende“, „Staat“ sowie „Import/Export“ verzeichnen, aufgestellt. Hierzu untergliedert Amemiya, ausgehend von der im vorherigen Kapitel geschätzten Größe der Gesamtbevölkerung, diese in die Gruppen „Arm“, „Reich“ sowie „Handel- und Gewerbetreibende“. Letztlich kommt er auf ein Bruttoinlandsprodukt in Höhe von 4430 Talenten.

Im dritten und letzten Teil werden zuerst die wirtschaftlichen Erkenntnisse von Xenophon geschildert. Darauf aufbauend gibt Amemiya einen allgemeinen Grundriss der Philosophie von Platon und Aristoteles, um anschließend näher auf die speziellen ökonomischen Denk- und Verhaltensmuster der beiden Philosophen einzugehen. Den Abschluss bildet eine Schilderung des Utilitarismus anhand der Arbeiten von Jeremy Bentham und John Stuart Mill, die zur Grundlage der modernen Wirtschaftswissenschaften wurden.

\footnotetext{
11 Hierbei schlich sich auf S. 57 ein kleiner Zahlendreher ein, denn nicht 1983, sondern 1893 argumentierte Karl Bücher, dass die griechische Ökonomie primitiver Natur sei. Vgl. zu dieser Thematik insgesamt H. Schneider, Die Bücher-Meyer Kontroverse, in: W. M. Calder III, A. Demandt (Hg.), Eduard Meyer. Leben und Leistung eines Universalhistorikers, Leiden 1990, S. 417-445, hier S. 419.

12 Vgl. I. Morris, The Athenian Economy Twenty Years after the Ancient Economy, in: Classical Philology 89 (1994), S. 351-366, hier S. 366.

${ }^{13}$ Weder im Text noch im Literaturverzeichnis findet sich ein Verweis auf P. Millett, Lending and borrowing in ancient Athens, Cambridge 1991.
} 
Zwei Karten des antiken Griechenland und Attikas, mehrere chronologische Übersichten der wichtigsten Ereignisse von 3300 v. Chr. bis 322 v. Chr., Angaben zu den wichtigsten Gewichts- und Maßeinheiten, ein Glossar griechischer Namen und Begriffe sowie ein Index runden das Buch ab.

Einerseits erleichtert die teilweise starke Untergliederung in einzelne, kleine Abschnitte einen zielorientierten Zugriff auf bestimmte Themengebiete und wird somit seinem Anspruch als Lehrbuch gerecht, andererseits kann es streckenweise zu einer Behinderung des Leseflusses führen. Auch wären eine ausführlichere Darlegung mancher Abschnitte und Gedanken sowie ein Schlusswort für eine stärkere Vergegenwärtigung des Stoffes dienlich gewesen. Gleichwohl ermöglichen zahlreiche Quellenzitate und Verweise auf Standardliteratur einen hohen Grad an Anschaulichkeit sowie einen schnellen Einstieg zu ausgewählten Sachgebieten. Trotz einer zum Teil idiosynkratischen Auswahl an Informationen zur Geschichte, Philosophie, Kultur und Gesellschaft des antiken Griechenland, welche nicht immer sinnvoll in Konnex mit dem System Wirtschaft gebracht werden können, zeichnet sich das Buch vor allem durch seine interdisziplinäre Herangehensweise aus. Aufgrund des ökonometrischen Zugriffs auf das antike Athen liefert Amemiya umfassende Erkenntnisse, welche für die Erforschung der antiken Wirtschaftsgeschichte einen großen Gewinn bedeuten. 